\title{
Molecular mimicry that primes for autoimmunity which is triggered by infection
}

\author{
Molecular Psychiatry (2002) 7, S32-S33. doi:10.1038/ \\ sj.mp.4001173
}

While the etiology and pathogenesis of autism are not known, several hypotheses have been proposed. These include a contribution by virus infection occurring early in life or in utero. Support for virus playing a role in this disease is the observation made by Chess. ${ }^{1,2}$ She found that a high proportion of children with congenital rubella had developed autism. Examination of patients years later led her to speculate that rubella virus was the primary etiologic agent. ${ }^{2}$ Subsequently, other viruses have been implicated or associated with autism. These include cytomegalovirus, ${ }^{3}$ parvovirus $B 19^{4}$ and more recently measles virus ${ }^{5-7}$ and human herpes virus. ${ }^{6}$

Another proposed mechanism or contributing factor may be an autoimmune response to the central nervous system (CNS) for autism. An increase in antibodies to the CNS proteins, myelin basic protein (MBP) and glial fibrillary acidic protein (GFAP) and neuron-axon filament protein have been seen in children with autism vs age-matched control subjects. ${ }^{8,9}$ These antibodies could affect the function of neurons leading to aberrant behavioral and social behaviors.

We have established a model where viruses can prime animals for autoimmune CNS disease by a mechanism of molecular mimicry. ${ }^{10}$ The autoimmune CNS disease can be triggered by a nonspecific immunological challenge. One hypothesis that could be tested by this experimental model is that virus infection early in life could imprint the immune repertoire such that at a later time a nonspecific infection could trigger an autoimmune event. ${ }^{10}$

To test this hypothesis, we made a cDNA (pUPLP) that encodes myelin proteolipid protein (PLP). In frame to the PLP coding region, the ubiquitin gene was inserted. Therefore, an ubiquinated PLP molecule would be synthesized within a transfected cell and targeted to the proteasomes for degradation and presentation. This would be similar to a viral infection where viral proteins are synthesized in the cytoplasm and targeted to proteasomes and presented by major histocompatibility antigen (MHC) class I molecules. Three week old (prior to puberty) SJL/J mice were then inoculated three times with pUPLP. The inoculated mice did not have any CNS changes and were by all appearances

Correspondence: RS Fujinami, Dept of Neurology, University of Utah School of Medicine, 30 N 1900 East, Rm 3R330, Salt Lake City, Utah 84132, USA. E-mail: Robert.Fujinami@hsc.utah.edu normal. When these mice were given a nonspecific immunologic challenge of complete Freund's adjuvant (CFA), $20 \%$ of the mice develop clinical signs and CNS lesions consistent with autoimmune CNS disease.

In the next set of experiments, we determined whether pUPLP could be replaced with a virus having molecular mimicry with a CNS protein. To this end, recombinant vaccinia viruses were constructed encoding PLP, myelin associated glycoprotein (MAG), or GFAP. Again young mice were infected with the different recombinant viruses having molecular mimicry with self CNS proteins or a control recombinant virus encoding $\beta$-galactosidase. The inoculated mice had no observable clinical signs or CNS pathology. At a later time mice were give a nonspecific immunologic challenge, CFA. The majority of mice previously infected with the recombinant viruses encoding the self proteins developed CNS lesions. None of the control virusinfected mice had any pathological changes in the CNS.

We next decided to investigate when a nonspecific viral infection could replace the CFA challenge. Therefore, young mice were inoculated three times with pUPLP and then rested. Mice were then infected with the recombinant virus encoding $\beta$-galactosidase. Mice were followed for the development of CNS autoimmune disease. Twenty percent of mice developed CNS lesions. ${ }^{10}$

Here we review our evidence that viruses can subclinically prime animals for CNS autoimmune disease that can be triggered by infection at a later time. We speculate that this may be one explanation for the difficulty in demonstrating an etiologic agent for autoimmune CNS disease such as multiple sclerosis. Should the etiology and pathogenesis of autism prove to be autoimmune and/or virus related, this might be a viable mechanism to explain its occurrence.

Three to four-week-old female SJL/J and PL/J mice were purchased from Jackson Laboratories (Bar Harbor, ME, USA). Mice were injected intramuscularly (i.m.) three times at one week intervals with $100 \mu \mathrm{g}$ plasmid DNA dissolved in $1 \mathrm{~N}$ saline. Mice were observed and weighed every other day. Seven days after the last injection, groups of mice received either PBS emulsified in complete Freund's adjuvant (CFA) or PBS alone subcutaneously (s.c.), at the base of the tail. Other control mice receiving DNA were 'challenged' with CFA alone. Mice were weighed daily after challenge.

In other experiments mice were primed with recombinant vaccinia viruses encoding PLP, ${ }^{11}$ MAG or GFAP $\left(5 \times 10^{6}\right.$ PFU i.p. of recombinant vaccinia virus) and 5 weeks later were challenged with CFA. 
The PLP gene was fused to ubiquitin-A76 and subcloned into the NotI site of plasmid pCMV, which was derived by excision of the $\beta$-galactosidase gene from pCMV $\beta$ (Clontech, Palo Alto, CA, USA). ${ }^{12}$ The resultant expression vector (pUPLP) contained the widely expressed immediate-early promoter from cytomegalovirus. Plasmid DNA was purified from transformed DH5 $\alpha$ Escherichia coli with an EndoFree Plasmid Maxi Kit (Qiagen, Chatsworth, CA, USA).

Histology and immunohistochemistry were performed as described in Tsunoda et al. ${ }^{13}$

\section{Acknowledgements}

The author would like to thank J Lindsay Whitton, MD, PhD, Fernando Rodriguez, Jane E Libbey, MS, Ikuo Tsunoda, MD, PhD, and Diethilde Theil for their work and input on this project, and Kathleen Borick for her help in preparation of the manuscript. This work was supported by NIH AI42525.

RS Fujinami

Department of Neurology, University of Utah School of Medicine, Salt Lake City, Utah, USA

1 Chess S. J Autism Child Schizophr 1971; 1: 33-47.

2 Chess S. J Autism Child Schizophr 1977; 7: 69-81.

3 Stubbs EG. J Autism Child Schizophr 1978; 8: 37-43

4 Anlar B et al. J Child Neurol 1994; 9: 104-105.

5 Singh VK et al. Clin Immunol Immunopathol 1998; 89: 105-108.

6 Wakefield AJ, Montgomery SM. Isr Med Assoc J 1999; 1: 183-187.

7 O’Leary JJ et al. Lancet 2000; 356: 772.

8 Singh VK et al. Brain Behav Immun 1993; 7: 97-103.

9 Singh VK et al. Pediatr Neurol 1997; 17: 88-90.

10 Theil DJ et al. J Neurovirol 2001; 7: 220-227.

11 Barnett LA et al. J Neuroimmunol 1993; 44: 15-25.

12 Tsunoda I et al. Brain Pathol 1999; 9: 481-493.

13 Tsunoda I et al. Brain Pathol 2000; 10: 402-418. 